\title{
Altered Glycolysis and Mitochondrial Respiration in a Zebrafish Model of Dravet Syndrome $1,2,3$
}

\author{
Maneesh G. Kumar, ${ }^{1}$ Shane Rowley, ${ }^{1}$ Ruth Fulton, ${ }^{1}$ Matthew T. Dinday, ${ }^{2}$ Scott C. Baraban, ${ }^{2}$ and \\ Manisha Patel ${ }^{1}$
}

DOI:http://dx.doi.org/10.1523/ENEURO.0008-16.2016

${ }^{1}$ Department of Pharmaceutical Sciences, University of Colorado Anschutz Medical Campus, Aurora, Colorado 80045, and ${ }^{2}$ Department of Neurological Surgery, University of California San Francisco, San Francisco, California 94143

\begin{abstract}
Altered metabolism is an important feature of many epileptic syndromes but has not been reported in Dravet syndrome (DS), a catastrophic childhood epilepsy associated with mutations in a voltage-activated sodium channel, Nav1.1 (SCN1A). To address this, we developed novel methodology to assess real-time changes in bioenergetics in zebrafish larvae between 4 and $6 \mathrm{~d}$ postfertilization (dpf). Baseline and 4-aminopyridine (4-AP) stimulated glycolytic flux and mitochondrial respiration were simultaneously assessed using a Seahorse Biosciences extracellular flux analyzer. Scn1Lab mutant zebrafish showed a decrease in baseline glycolytic rate and oxygen consumption rate (OCR) compared to controls. A ketogenic diet formulation rescued mutant zebrafish metabolism to control levels. Increasing neuronal excitability with 4-AP resulted in an immediate increase in glycolytic rates in wild-type zebrafish, whereas mitochondrial OCR increased slightly and quickly recovered to baseline values. In contrast, scn1Lab mutant zebrafish showed a significantly slower and exaggerated increase of both glycolytic rates and OCR after 4-AP. The underlying mechanism of decreased baseline OCR in scn1Lab mutants was not because of altered mitochondrial DNA content or dysfunction of enzymes in the electron transport chain or tricarboxylic acid cycle. Examination of glucose metabolism using a PCR array identified five glycolytic genes that were downregulated in scn1Lab mutant zebrafish. Our findings in scn1Lab mutant zebrafish suggest that glucose and mitochondrial hypometabolism contribute to the pathophysiology of DS.
\end{abstract}

Key words: Dravet syndrome; epilepsy; glycolysis; metabolism; mitochondrial respiration; zebrafish

\section{Significance Statement}

These studies demonstrate that metabolism can be studied in zebrafish, and that this novel approach can be used: (1) to evaluate chemoconvulsant or genetic zebrafish models of epilepsy, or (2) in metabolismbased drug screening efforts to identify compounds that modulate glycolysis or mitochondrial function. As more models of epilepsy become available, the array of techniques demonstrated here can be used to rapidly characterize metabolic contributions to disease states.

\section{Introduction}

Glycolysis and mitochondrial oxidative phosphorylation are key energy producing pathways in the brain. These

Received January 12, 2016; accepted March 24, 2016; First published March 29, 2016.

${ }^{1}$ The authors report no conflict of interest.

${ }^{2}$ Author contributions: M.G.K., S.C.B., and M.P. designed research; M.G.K. performed research; M.G.K. analyzed data; S.R., R.F., M.T.D., and S.C.B. contributed unpublished reagents/analytic tools; M.G.K., S.C.B., and M.P. wrote the paper.
${ }^{3}$ This work was supported by the NIH R01NS39587(M.P.), NIH R01NS079214 (S.C.B.), and (in part) by a sabbatical grant from the Associate Dean of Research Seed Grant Program, Skaggs School of Pharmacy and Pharmaceutical Sciences, University of Colorado. We thank the zebrafish transgenic core at the University of Colorado (P30NS048154), in particular Dr Kristin Artinger, Ana-Laura Hernandez, Morgan Singleton, and Michelle Tellez.

Correspondence should be addressed to Dr Manisha Patel, University of Colorado, Skaggs School of Pharmacy and Pharmaceutical Sciences, Mail Stop C238 V20-3119, 12850 East Montview Boulevard, Aurora, CO 80045. E-mail: manisha.patel@ucdenver.edu. 
metabolic pathways may play a role in the control of seizures and epileptogenesis (Rowley et al., 2015). For example, glycolytic rates are acutely increased during ictal activity (Stafstrom et al., 2008) and ictal hypermetabolism in human epileptic foci is followed by interictal hypometabolism, probably resulting from a decreased mitochondrial bioenergetic capacity (Chugani et al., 1994; Lee et al., 2012). Hypometabolism associated with seizures may reflect glucose transport abnormalities or defects in electron transport chain (ETC) enzymes (Tenney et al., 2014). Seizures are also associated with an imbalance in ATP levels (Grisar, 1984) and represent a common symptom in patients with mitochondrial disease (Wallace et al., 1988; Mecocci et al., 1993; Liang et al., 2012).

Although altered metabolism may be a feature of acquired and/or focal epilepsies, it has not been systematically investigated in any genetic form of epilepsy. Dravet syndrome (DS) is one example of a severe genetic epilepsy most commonly associated with de novo mutations in a brain-specific voltage-activated sodium channel (SCN1A). DS children exhibit significant developmental delays, cognitive deficits, behavioral disturbances, and increased risk of sudden unexpected death in epilepsy (SUDEP; Dravet, 2011). Two types of observations suggest that metabolic dysfunction may be occurring in DS. First, mitochondrial defects in muscle biopsies have been reported in patients. Second, some DS children respond positively to treatment with ketogenic diets (KDs; Caraballo, 2011). Although numerous mechanisms may underlie the efficacy of KDs (Gano et al., 2014), these observations suggest that energy metabolism is not well studied in DS, or any form of genetic epilepsy. Here we developed novel techniques to show, for the first time, that glycolysis and mitochondrial respiration are abnormal in a zebrafish model of DS and its rescue by a form of a KD. Moreover, altered metabolism in scn1Lab mutants was accompanied by downregulation of several glycolytic genes rather than defects in select mitochondrial enzyme activities.

\section{Materials and Methods}

\section{Animal care}

Scn1Lab mutant zebrafish were obtained from the Baraban laboratory at the University of California San Francisco (UCSF) and bred in the University of Colorado Anschutz Medical Campus (UCD) zebrafish core facility. When obtained from UCSF, eggs were shipped overnight and immediately placed in a $28.5^{\circ} \mathrm{C}$ incubator upon arrival. Zebrafish larvae were maintained in "embryo medium" consisting of $0.03 \%$ Instant Ocean (Aquarium Systems) in deionized water containing $0.2 \mathrm{ppm}$ methylene blue as a fungicide with no additional glucose anapleurotic substrates added. Homozygous mutants (sorted based on pigmentation) and age-matched sibling larvae

DOI:http://dx.doi.org/10.1523/ENEURO.0008-16.2016

Copyright (๑) 2015 Kumar et al.

This is an open-access article distributed under the terms of the Creative Commons Attribution 4.0 International, which permits unrestricted use, distribution and reproduction in any medium provided that the original work is properly attributed. were used at 4-6 d postfertilization (dpf). Pentylenetetrazole (PTZ; Sigma-Aldrich) was dissolved in embryo medium, $\mathrm{pH}$ balanced to 7.4 , and bath applied. KD water was prepared by sonication of $200 \mu \mathrm{M}$ palmitate (SigmaAldrich) and laurate (Sigma-Aldrich) in embryo media containing $100 \mu \mathrm{M}$ phosphatidyl choline (Sigma-Aldrich) and bath applied (Taylor et al., 2004).

\section{Metabolic measurements}

Glycolysis and mitochondrial respiration rates were simultaneously measured in live zebrafish in an XF24 or XF24e analyzer (Seahorse Bioscience). One fish was loaded per well of a 24-well islet plate and mesh screen placed to hold the zebrafish in place. 4-AP (SigmaAldrich) was prepared in embryo medium at a stock concentration of $40 \mathrm{~mm}$ and $\mathrm{pH}$ balanced to $\sim 7.4$. 4-AP was injected by the XF24 analyzer at a final concentration of $4 \mathrm{~mm}$.

\section{Behavioral seizure analysis}

Zebrafish were placed individually in 96-well Falcon culture dishes. Each well contained $\sim 75 \mu$ lembryo media and one $5 \mathrm{dpf}$ WT zebrafish larvae. Swim behavior was monitored in a DanioVision system, as described previously in the literature (Baraban et al., 2013). Recording sessions (2 $\mathrm{min}$ ) were analyzed off-line and scored for seizure stage (Baraban et al., 2005) by an investigator blind to the status of the fish.

\section{Mitochondrial copy number}

Relative mitochondrial copy number was determined by real-time PCR (Hunter et al., 2010; Artuso et al., 2012). DNA was extracted from individual fish using the DNeasy Blood and Tissue Kit (Qiagen) following the manufacturersupplied instructions. A short section of mitochondrial DNA was amplified using Power SYBR green (Forward: 5'-CAAACACAAGCCTCGCCTGTTTAC-3'; Reverse: 5'CACTGACTTGATGGGGGAGACAGT-3'). This was normalized to the nuclear gene polg1 (Forward: 5 '-GAGAGC GTCTATAAGGAGTAC-3'; Reverse: 5' - GAGCTCATCAGA AACAGGACT-3'). Primers were ordered from Integrated DNA Technologies (Coralville). Fish were exposed to 4-AP for $1 \mathrm{~h}$.

\section{Enzyme assays}

Activity of complexes I-IV was determined from total protein isolates from 25 to 30 pooled zebrafish embryos at 4-6 dpf. Total protein was isolated by resuspending zebrafish in PBS with $0.01 \%$ Triton-X and protease inhibitors followed by probe sonication at $30 \%$ intensity for three pulses of $5 \mathrm{~s}$ each. Lysate was centrifuged at 10,000 rpm for $5 \mathrm{~min}$ to remove insoluble material. Total protein concentration was determined by Bradford assay. Complex activity was determined by UV spectrophotometry (Ma et al., 2011). Complex I was determined by the oxidation of NADH at $340 \mathrm{~nm}$. The assay solution consisted of $25 \mathrm{~mm}$ potassium phosphate, $5 \mathrm{~mm} \mathrm{MgCl}_{2}, 2 \mathrm{~mm} \mathrm{KCN}$, $2.5 \mathrm{mg} / \mathrm{ml} \mathrm{BSA}, 0.13 \mathrm{~mm} \mathrm{NADH}, 2 \mathrm{ug} / \mathrm{ml}$ antimycin A, and $65 \mu \mathrm{M}$ ubiquinone1 equilibrated to $30^{\circ} \mathrm{C}$. Forty micrograms of total protein was added and activity measured for 5 min. Nonspecific activity was determined by addition 
of $2 \mu \mathrm{g} / \mathrm{ml}$ of rotenone prior to protein addition. Complex II was determined by reduction of dichlorophenol indophenols at $600 \mathrm{~nm}$. The assay solution consisted of $25 \mathrm{~mm}$ $\mathrm{KPO}_{4}, 5 \mathrm{~mm} \mathrm{MgCl} 2,2 \mathrm{~mm} \mathrm{KCN}, 20 \mathrm{~mm}$ sodium succinate, $50 \mu \mathrm{M}$ dichlorophenol indophenol, $2 \mu \mathrm{g} / \mathrm{ml}$ rotenone, and $2 \mu \mathrm{g} / \mathrm{ml}$ antimycin A. After equilibrating to $30^{\circ} \mathrm{C}, 40 \mu \mathrm{g}$ of total protein was added along with $65 \mu \mathrm{m}$ ubiquinone 1 to start the reaction. Activity was determined between 3 and 5 min. Complex III was determined by the reduction of cytochrome $C$ at $550 \mathrm{~nm}$. The assay solution consisted of $50 \mathrm{~mm} \mathrm{KPO}_{4}$, $1 \mathrm{~mm} N$-dodecyl- $\beta$-D-maltoside, $2 \mathrm{~mm} \mathrm{KCN}$, and $20 \mathrm{~mm} \mathrm{NADH}$ at $30^{\circ} \mathrm{C}$. Forty micrograms of total protein was added along with $100 \mu \mathrm{M}$ of reduced decylbenzylquinone and $40 \mu \mathrm{M}$ oxidized cytochrome $\mathrm{C}$ to start the reaction. Activity was measured for $3 \mathrm{~min}$. Complex IV was determined by oxidation of cytochrome $C$ at $550 \mathrm{~nm}$. The assay solution consisted of $20 \mathrm{mM} \mathrm{KPO}_{4}$ and $0.45 \mathrm{~mm}$ $\mathrm{N}$-dodecyl- $\beta$-D-maltoside equilibrated to $30^{\circ} \mathrm{C}$. Forty micrograms of total protein and reduced cytochrome $\mathrm{C}$ was added to a final concentration of $15 \mu \mathrm{M}$ and activity measured for 3 mins.

Activity of select enzymes of the tricarboxylic acid (TCA) cycle, aconitase, fumarase, and malate dehydrogenase, was determined from total protein isolates from 30 pooled zebrafish embryos at $5 \mathrm{dpf}$. Total protein was isolated as above with the exception of probe sonication at $30 \%$ intensity for two pulses of $1 \mathrm{~s}$ each. Aconitase and fumarase activity was determined by UV spectrophotometry by following the dehydration of DL-isocitrate or L-malate, respectively, at $240 \mathrm{~nm}$ (Patel et al., 1996). The aconitase assay buffer consisted of $50 \mathrm{mM}$ Tris $\mathrm{HCl}$ and $600 \mu \mathrm{M}$ $\mathrm{MnCl}_{2}$ at $\mathrm{pH}$ 7.4. DL-Isocitrate substrate was added to a final concentration of $20 \mathrm{~mm}$ along with $20 \mu \mathrm{l}$ of total protein. Activity was measured for $3 \mathrm{~min}$. Aconitase activity was inhibited by addition of $0.1 \mathrm{~mm}$ potassium ferricyanide. Fumarase assay buffer consisted of $30 \mathrm{~mm}$ $\mathrm{KH}_{2} \mathrm{PO}_{4}$ and $100 \mu \mathrm{M}$ EDTA at a pH of 7.4. L-Malate was added to a final concentration of $5 \mathrm{~mm}$ along with $20 \mu \mathrm{l}$ of total protein. Activity was measured for $3 \mathrm{~min}$. Fumarase activity showed stereospecificity for L-malate; D-malate was inactive. Malate dehydrogenase was determined by measuring the decrease in absorbance at $340 \mathrm{~nm}$ resulting from oxidation of NADH. The assay buffer consisted of $0.1 \mathrm{M} \mathrm{KPO}_{4}, 200 \mu \mathrm{M}$ oxaloacetic acid, and $250 \mu \mathrm{M} \mathrm{NADH}$, $\mathrm{pH}$ 7.4. Five microliters of total protein was added and activity measured for $5 \mathrm{~min}$. All TCA cycle enzyme assays were performed at room temperature.

\section{Gene expression}

Six to eight zebrafish were pooled for each group. RNA was extracted by TRIzol $1 \mathrm{~h}$ after 4-AP treatment. cDNA was synthesized using the $\mathrm{RT}^{2}$ First Strand Kit (SABiosciences) and hybridized to the Glucose Metabolism PCR array (SABiosciences). Array was analyzed using Applied Biosystem 7500 Real-Time PCR System. Data was analyzed using SABiosciences' on-line data analysis suite (http://www.sabiosciences.com/pcrarraydataanalysis. php). Array results were verified using specific Taqman primers (Applied Biosystems). Fish were exposed to 4-AP for $1 \mathrm{~h}$.

\section{Statistics}

Statistical methods were performed with GraphPad Prism v6.04 for Windows. Analyses include Holm-Sidak $t$ test (for baseline comparisons) and two-way one-way ANOVA with Tukey's multiple-comparisons test. Behavioral seizure data were analyzed by Shapiro-Wilk normality test followed by Kruskal-Wallis ANOVA on Ranks with Tukey's multiple comparison post hoc test.

\section{Results}

To measure real-time glycolytic and mitochondrial respiration rates live zebrafish (4-6 dpf) were placed in an extracellular flux analyzer (Seahorse Biosciences). The analyzer measures extracellular acidification rate (ECAR) and oxygen consumption rate (OCR) in a transient microchamber over $3 \mathrm{~min}$, representing glycolysis and mitochondrial respiration, respectively (Wu, 2009). Baseline glycolysis rates in scn1Lab mutant and age-matched WT zebrafish were $7.70 \pm 0.18 \mathrm{mpH} / \mathrm{min}$ and $13.06 \pm 0.61$ $\mathrm{mpH} / \mathrm{min}$ (Fig. $1 A$; Table 1), respectively, indicating a $41 \%$ decrease $(p<0.0001)^{a}$ of baseline glycolysis. Similarly, basal OCR was significantly decreased in scn $1 \mathrm{Lab}$ mutant zebrafish (189.3 $\pm 3.47 \mathrm{pmol} / \mathrm{min})$ compared with WT controls $\left(296.5 \pm 1.929 \mathrm{pmol} / \mathrm{min}, p<0.0001^{\mathrm{b}}\right.$; Fig. $\left.1 B\right)$. Zebrafish obtained from UCSF or bred at UCD and metabolism measured using the Seahorse XF24 or an updated version, the XF24e, showed similar alterations in metabolism between scn1Lab and WT siblings (see Fig. 6).

Scn1Lab mutant zebrafish have been reported to have seizures very early in life, starting at about $3 \mathrm{dpf}$ (Baraban et al., 2013). To determine the consequences of increased neuronal excitability associated with induced seizure activity, WT zebrafish were stimulated with 4-AP and glycolytic and mitochondrial respiration rates were measured (Figs. 1A,B). 4-AP, a chemoconvulsant previously shown to induce electrographic seizures in zebrafish (Baraban et al., 2007), increases metabolic demand in cortical cells and synaptosomes (Tibbs et al., 1989; Flynn et al., 2011). Following metabolic challenge with a single, high dose of 4-AP, WT zebrafish showed increased behavioral seizure activity (Fig. 1C,D) and 360\% increase in glycolysis within 8 min. Scn1Lab mutants, which exhibit spontaneous seizures at a rate of $\sim 1$ ictal-like event per minute (Baraban et al., 2013), also showed increased glycolytic rate after 4-AP stimulation albeit in a delayed manner, achieving maximal glycolytic rates about 30 min after 4-AP addition (Fig. 1A). In scn1Lab mutant zebrafish, glycolysis was increased $350 \%$ from baseline to an average of $27.26 \pm$ $1.12 \mathrm{mpH} / \mathrm{min}\left(p<0.0001^{\mathrm{e}}\right.$, Fig. $\left.1 A\right)$. Both the immediate and delayed increase of glycolytic rates in WT and scn1Lab mutant zebrafish were sustained for the duration of the experiment, $48 \mathrm{~min}$. After 24-32 min when scn1Lab mutant zebrafish had maximal response to 4-AP, the fold-change was significantly greater in scn1Lab mutant zebrafish than that of WT zebrafish. Behavioral seizure analysis on an independent clutch of WT larvae demonstrated that a single concentration of $4 \mathrm{~mm} 4-\mathrm{AP}$ induced the severe convulsive seizure behavior (Stage III) at the 8 min time point in 23 of 48 larvae; circling Stage II behavior 
A

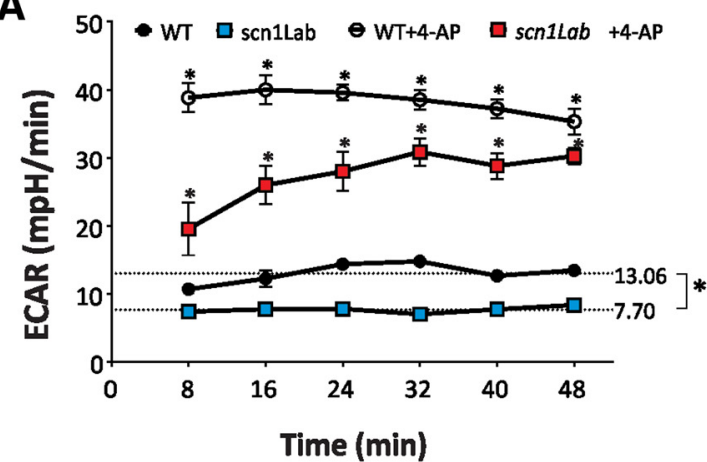

C

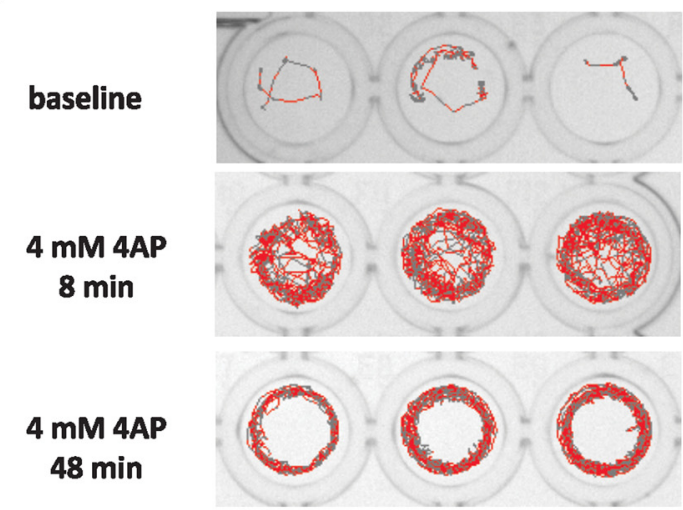

B

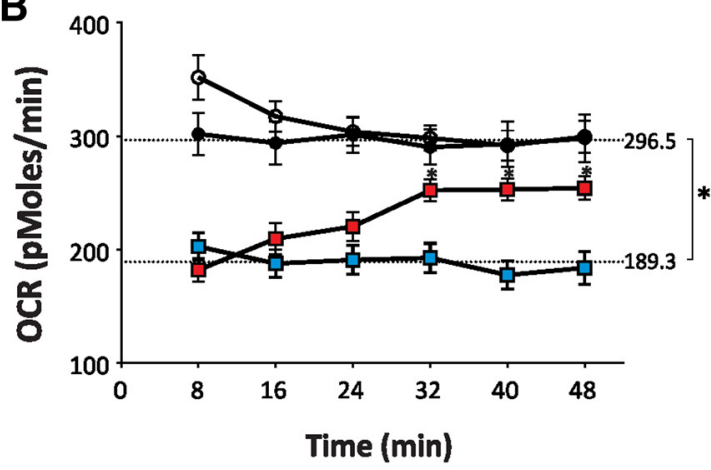

D

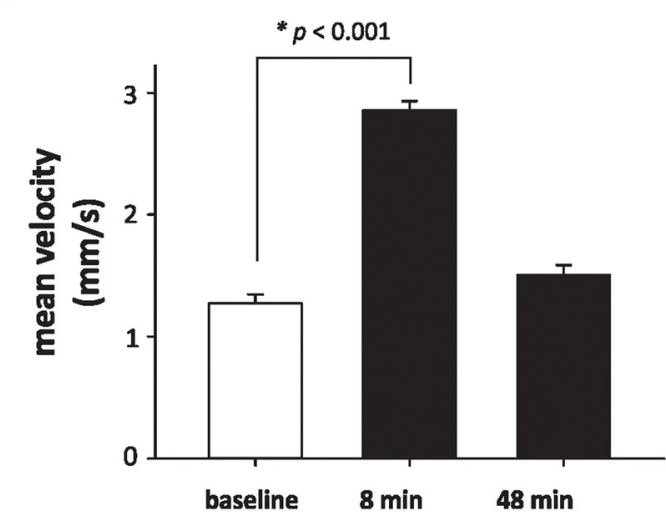

Figure 1. Glycolytic and mitochondrial respiration rates in WT and scn1Lab mutant zebrafish at baseline and after 4-AP stimulation. $\boldsymbol{A}$, B, Scn1Lab mutant zebrafish have lower baseline glycolytic and mitochondrial respiration rates than WT zebrafish. 4-AP immediately increases glycolytic and mitochondrial respiration rates in WT zebrafish. Scn1 Lab mutant zebrafish have a delayed response to 4-AP of $\sim 30$ min. Statistical analysis shows changes relative to time-matched untreated controls; points represent means \pm S.E.M. $N=14$ (WT), 16 (scn1Lab), 16 (WT+4-AP), 14 (scn1Lab+4-AP) individual animals, mean \pm S.E.M. $\boldsymbol{A}$, WT vs scn1Lab: $p<0.0001^{\mathrm{a}}$; WT vs WT+4-AP, $p=$ $2.38 \mathrm{e}-30(8 \mathrm{~min})^{\mathrm{o}}, p=8.41 \mathrm{e}-30(16 \mathrm{~min})^{\mathrm{p}}, p=3.50 \mathrm{e}-26(24 \mathrm{~min})^{\mathrm{q}}, p=3.99 \mathrm{e}-24(32 \mathrm{~min})^{\mathrm{r}}, p=2.92 \mathrm{e}-25(40 \mathrm{~min})^{\mathrm{s}}, p=1.70 \mathrm{e}-21(48 \mathrm{~min})^{\mathrm{t}}$ scn1Lab vs scn1Lab+4-AP: $p=3.28 \mathrm{e}-6(8 \mathrm{~min})^{\mathrm{u}}, p=1.65 \mathrm{e}-11(16 \mathrm{~min})^{\mathrm{v}}, p=1.86 \mathrm{e}-13(24 \mathrm{~min})^{\mathrm{w}}, p=3.08 \mathrm{e}-17(32 \mathrm{~min})^{\mathrm{x}}, p=2.34 \mathrm{e}-14$ $(40 \mathrm{~min})^{\mathrm{y}}, p=3.52 \mathrm{e}-15(48 \mathrm{~min})^{\mathrm{z}}$. $\boldsymbol{B}$, WT vs scn1La: $p<0.0001^{\mathrm{aa}}$; scn1Lab versus scn1Lab+4-AP: $p=0.00071(32 \mathrm{~min})^{\mathrm{bb}}, p=2.20 \mathrm{e}-5$ $(40 \mathrm{~min})^{\mathrm{cC}}, p=7.24 \mathrm{e}-5(48 \mathrm{~min})^{\mathrm{dd}}$. C, $\boldsymbol{D}$, Locomotion plots for behavioral seizure activity in WT zebrafish exposed to $4 \mathrm{mmm} 4-\mathrm{AP}$. Bar plot showing the mean \pm SEM for WT fish at baseline, 8 min after exposure to 4-AP and 48 min after exposure to 4 -AP. $N=48 \mathrm{WT}$ fish; Kruskal-Wallis one-way ANOVA on ranks with a post hoc Tukey test. $p<0.05^{\mathrm{c}, \mathrm{d}}$ (8 min vs baseline).

was noted in 25 of 48 larvae. After continuous exposure to 4-AP, seizure behavior corresponding to Stage II was observed in the majority of zebrafish larvae (47 of 48). Representative locomotion plots are shown for WT zebrafish at baseline and following exposure to $4 \mathrm{~mm}$ 4-AP (Fig. 1C). Mean velocity measurements, which act as a surrogate marker for Stage III behavior (Baraban et al., 2013) were significantly increased at the 8 min exposure point (Fig. 1D ${ }^{\mathrm{C}}$ ).

WT zebrafish showed a small, nonsignificant, increase in mitochondrial respiration immediately after 4-AP stimulation, which rapidly returned to baseline (Fig. 1B). However, scn1Lab mutant zebrafish showed a delayed and gradual increase in mitochondrial respiration with maximal increase about 30 min after stimulation. Mitochondrial respiration was increased $\sim 142 \%$ from baseline to 253.1 $\mathrm{pmol} / \mathrm{min}$ in scn1Lab mutant compared with WT zebrafish.

These data, when plotted as OCR versus ECAR, show a unique metabolic shift (Fig. 2A). At baseline, WT ze- brafish are more glycolytic and oxidative than mutant zebrafish, but both processes are in a narrow metabolic field. Increased neuronal excitability shifts the metabolic field toward the glycolytic direction. The mutant zebrafish increase glycolysis and oxidative phosphorylation after 4-AP exposure to approach the metabolic state of WT zebrafish after 4-AP. This suggests that mutant zebrafish retain a similar metabolic capacity as WT zebrafish, or in other words, there are no defective enzymes in the downstream energetic pathway. To confirm this, we assayed three different parameters. To ensure that alterations in mitochondrial OCR were not due to differences in mitochondrial content, relative mitochondrial copy number was determined. Results demonstrate that there is no significant difference between total mitochondrial copy numbers between groups ( $p=.2056^{\mathrm{h}}$; Fig. $2 B$ ). Next, to determine whether OCR differences were due to alterations in ETC activity, complexes I-IV were assayed in WT and scn1Lab mutant zebrafish. No differences were observed in the activity of any of the complexes $\left(p=0.9367^{\mathrm{i}}\right.$; 
Table 1. Statistical table

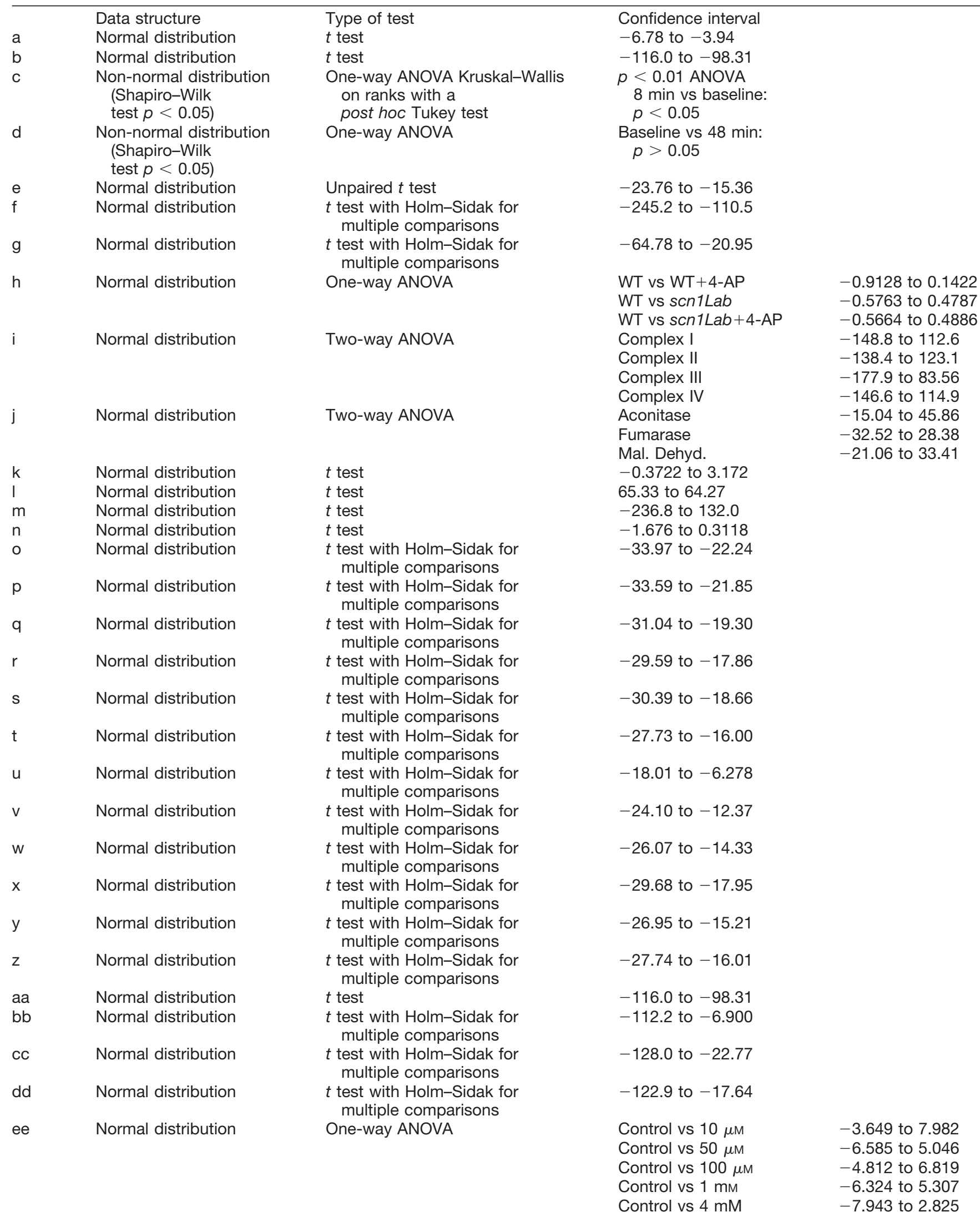


Table 1. (Continued)

\begin{tabular}{|c|c|c|c|c|}
\hline & Data structure & Type of test & Confidence interval & \\
\hline \multirow[t]{5}{*}{$\mathrm{ff}$} & Normal distribution & One-way ANOVA & Control vs $10 \mu \mathrm{M}$ & -67.77 to 93.79 \\
\hline & & & Control vs $50 \mu \mathrm{M}$ & -62.06 to 99.49 \\
\hline & & & Control vs $100 \mu \mathrm{M}$ & -39.08 to 122.5 \\
\hline & & & Control vs $1 \mathrm{~mm}$ & -19.22 to 142.3 \\
\hline & & & Control vs $4 \mathrm{~mm}$ & -19.85 to 129.7 \\
\hline \multirow[t]{3}{*}{ gg } & Normal distribution & One-way ANOVA & WT vs WT+4-AP & -0.9128 to 0.1422 \\
\hline & & & WT vs scn1Lab & -0.5763 to 0.4787 \\
\hline & & & WT vs scn1Lab+4-AP & -0.5664 to 0.4886 \\
\hline \multirow[t]{4}{*}{$\mathrm{hh}$} & Normal distribution & Two-way ANOVA & Complex I & -148.8 to 112.6 \\
\hline & & & Complex II & -138.4 to 123.1 \\
\hline & & & Complex III & -177.9 to 83.56 \\
\hline & & & Complex IV & -146.6 to 114.9 \\
\hline \multirow[t]{3}{*}{ ii } & Normal distribution & Two-way ANOVA & Aconitase & -15.04 to 45.86 \\
\hline & & & Fumarase & -32.52 to 28.38 \\
\hline & & & Mal. Dehyd. & -21.06 to 33.41 \\
\hline jj & Normal distribution & $t$ test & 1.783 to 8.531 & \\
\hline $\mathrm{kk}$ & Normal distribution & $t$ test & -4.128 to -2.727 & \\
\hline II & Normal distribution & $t$ test & -36.51 to -22.87 & \\
\hline $\mathrm{mm}$ & Normal distribution & $t$ test & -98.59 to -32.08 & \\
\hline $\mathrm{nn}$ & Normal distribution & $t$ test & -104.4 to -49.14 & \\
\hline O० & Normal distribution & $t$ test & -46.07 to -30.43 & \\
\hline $\mathrm{pp}$ & Normal distribution & $t$ test & -52.57 to -32.94 & \\
\hline qq & Normal distribution & $t$ test & -35.93 to -11.69 & \\
\hline $\mathrm{rr}$ & Normal distribution & $t$ test & -32.95 to -15.50 & \\
\hline
\end{tabular}

Fig. 2C). OCR may also have been decreased in scn1Lab mutant zebrafish if the TCA cycle enzymes were inactive, providing fewer electron donors for the ETC. Analysis of aconitase, fumarase, and malate dehydrogenase demonstrated no difference in enzymatic activity in WT and mutant zebrafish ( $p=0.5801^{j}$; Fig. $2 D$ ). This suggests that the primary metabolic defect may lie even further upstream, in glycolysis.

We therefore focused on the glycolytic pathway to understand the mechanistic basis of altered metabolism in scn1Lab mutant zebrafish. Since the activity of oxidative phosphorylation enzymes were unaltered in scn1Lab mutants and previous results suggested that genes related to metabolic processes are differentially expressed in scn1Lab mutant zebrafish (Baraban et al., 2007), we examined gene expression changes using an 84 probe glucose metabolism microarray (Fig. $3 A, B$ ). Of these, six genes were changed at least twofold (Fig. $3 C$ ). Five of these six were downregulated in scn1Lab mutant zebrafish compared with WT and included g6pca.1, pck1, pck2, pdk2, and phkg1a. Gene expression was also analyzed $1 \mathrm{~h}$ after 4-AP treatment. In 4-AP stimulated zebrafish, four genes were changed at least twofold compared with untreated controls (gck, pck1, pdk2, and pdk4). PCR array results were verified using specific Taqman primers, which were available for pck1, pck2, and $p d k 2$. PCR results verified fold-changes of $p c k 1(-2.83 \pm$ $0.45)$, pck2 (-2.36 \pm 0.45$)$, and pdk2 $(-2.23 \pm 0.42)$ in scn1Lab versus WT zebrafish (Fig. $3 C$, inset). WT zebrafish after 4-AP stimulation were also verified to have a $2.08 \pm 0.48$-fold increase in pdk2 expression; scn1Lab mutant zebrafish had a fold-change of $15.16 \pm 4.93$ in pdk2 expression (Fig. 3C, inset). These results suggest that baseline decreases in ECAR and OCR in scn1Lab mutants likely results from mutation-induced downregulation of key glycolytic genes leading to an overall decreased flux of substrates through these pathways. Exaggerated responses in ECAR and OCR in scn1Lab mutants could be the result of mutation-induced upregulation of key glycolytic genes (ie, pck1 and pdk2). Together, alterations in glycolytic gene expression provide a mechanistic basis for ion channel related alterations in metabolism.

Finally, to determine whether metabolic deficits could be rescued, we exposed developing zebrafish larvae to a modified KD (Taylor et al., 2004) for 48 h. In scn1Lab mutant zebrafish, baseline metabolism was raised to levels similar to WT controls for both glycolysis $\left(p=0.1043^{\mathrm{k}}\right)$ and respiration ( $p=0.9853^{\prime}$; Fig. $4 A$ ). 4-AP treatment produced a similar magnitude of increase in ECAR in both vehicle and KD-exposed WT controls $\left(p=0.4744^{\mathrm{m}}\right)$; however, the response to 4-AP was suppressed in the KDexposed scn1Lab mutant zebrafish to the same rates as WT zebrafish ( $p=0.1294^{\text {n; }}$ Fig. $4 B$ ).

\section{Discussion}

Here we demonstrate significant decreases in metabolism in a zebrafish model of SCN1A-related epilepsy. These mutants exhibit hyperactivity, including convulsive behavior, spontaneous electrographic seizures, shortened lifespan and a pharmacological profile similar to the human condition (Baraban et al., 2013; Dinday and Baraban, 2015). We now demonstrate, using novel methodology, that these scn1Lab mutant zebrafish, representing one example of a genetic epilepsy, exhibit decreased glycolytic and mitochondrial respiration rates. Although mitochondrial respiration has been measured in early embryogenesis up to $48 \mathrm{~h}$ postfertilization (Stackley et al., 
A

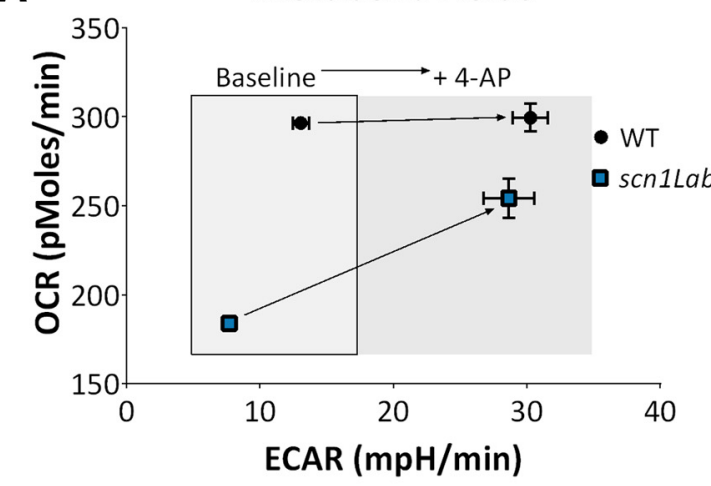

C

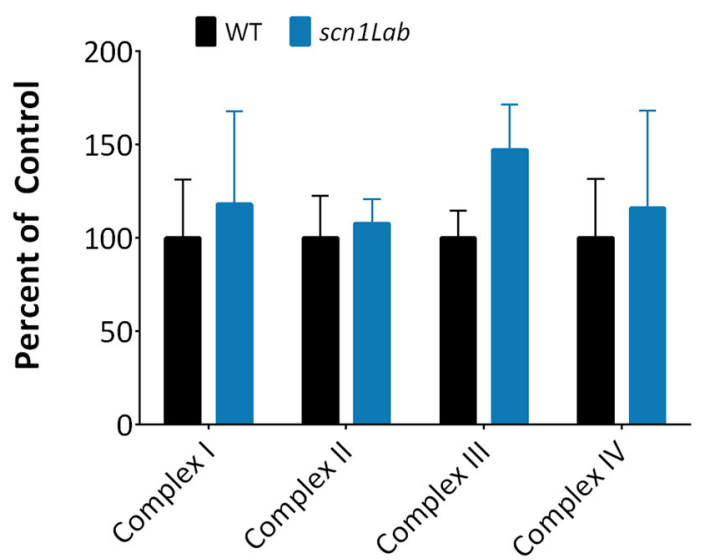

B

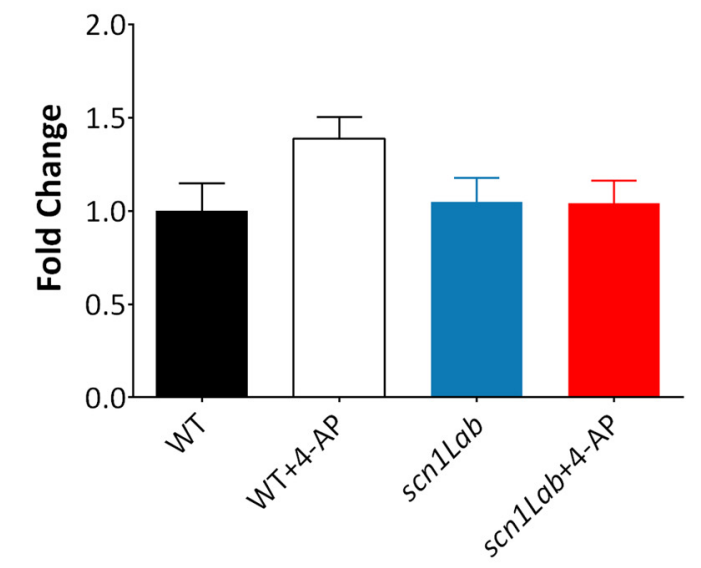

D

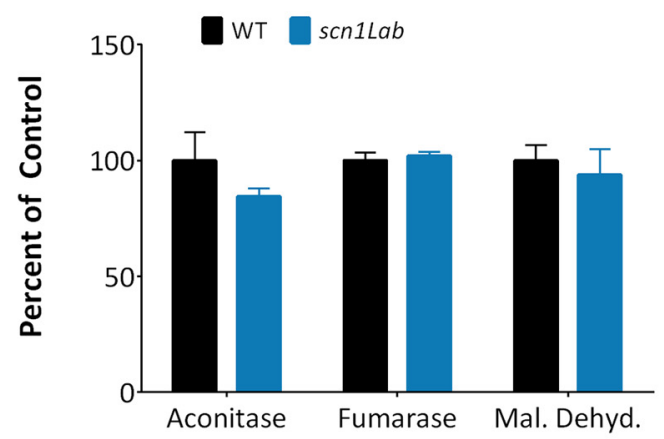

Figure 2. Respiratory chain complex activity in WT and scn1Lab mutant zebrafish. $\boldsymbol{A}$, ECAR and OCR from Figure 1 are replotted to demonstrate the metabolic field and the increases in metabolic field after treatment with 4-AP. Scn1Lab mutant zebrafish increase metabolism to approach the metabolic state of WT zebrafish after 4-AP, suggesting mutant zebrafish retain a similar metabolic capacity as WT zebrafish; each point represents mean \pm S.E.M. B, Relative mitochondrial copy number was determined by total mitochondrial DNA. No significant differences were found ( $n=3$ individual animals per group; one-way ANOVA, $\left.p=0.2056^{99}\right)$. $C$, There is no difference in activity in complexes I-IV in WT and scn1Lab mutant zebrafish. Bars represent the mean \pm SEM relative to WT activity, $\mathrm{n}=3$ groups with 25-30 fish pooled per group. Two-way ANOVA, interaction, $p=0.9367^{\text {hh }}$. $\boldsymbol{D}$, There are no differences in activity in selected TCA cycle enzymes in WT and scn1Lab mutant zebrafish. Bars represent the mean \pm SEM relative to WT activity, $n=4$ groups with 30 fish per group. Two-way ANOVA, interaction, $p=0.5801^{i i}$.

2011) and cell-based (Giménez-Cassina et al., 2012) or rodent-based (Rowley et al., 2015) systems, this is the first time metabolism has been measured in live zebrafish larvae and could be useful in identifying metabolic dysfunction in the growing armamentarium of zebrafish mutants.

Hypometabolism in DS has not been extensively reported in the literature. However, in a single study evaluating the metabolic status of patients with DS, Craig et al. (2012) showed comorbid electron transport defects in two patients. One had impaired complex III activity, and the other complex IV, in muscle biopsies from two DS patients. The etiology of the comorbidity is not entirely obvious but the authors suggest an scn1a mutation is not the only cause. Our results support these suppositions.
The scn1 Lab mutant zebrafish did not show any defects in ETC complexes I-IV, suggesting respiratory chain defects may not be inherent to DS.

The mechanism by which loss of sodium-channel function results in the constellation of symptoms observed in DS is incompletely understood. Our results suggest a general decrease in expression of glycolysis related genes resulting in decreased glycolytic substrates driving mitochondrial respiration. It is possible seizures, which are spontaneously occurring in these mutants, induce oxidative stress resulting in posttranslational oxidative modification and decreased activities of complex I (Ryan et al., 2012). Metabolic dysfunction, in particular mitochondrial dysfunction, has been known to have epilepsy as a dominant or collateral feature of the phenotype (Fin- 
A

B

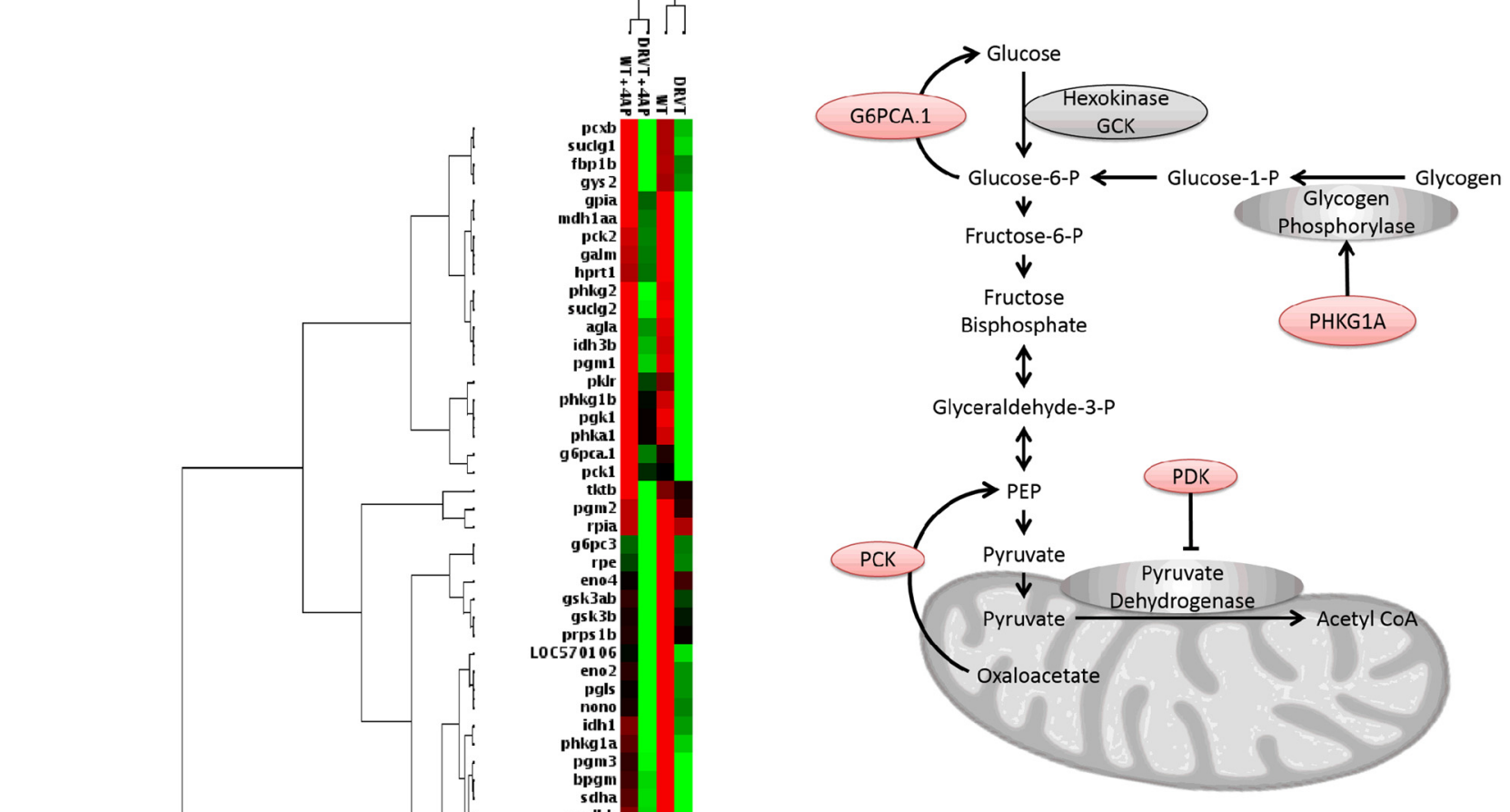

C

\section{Gene Expression}

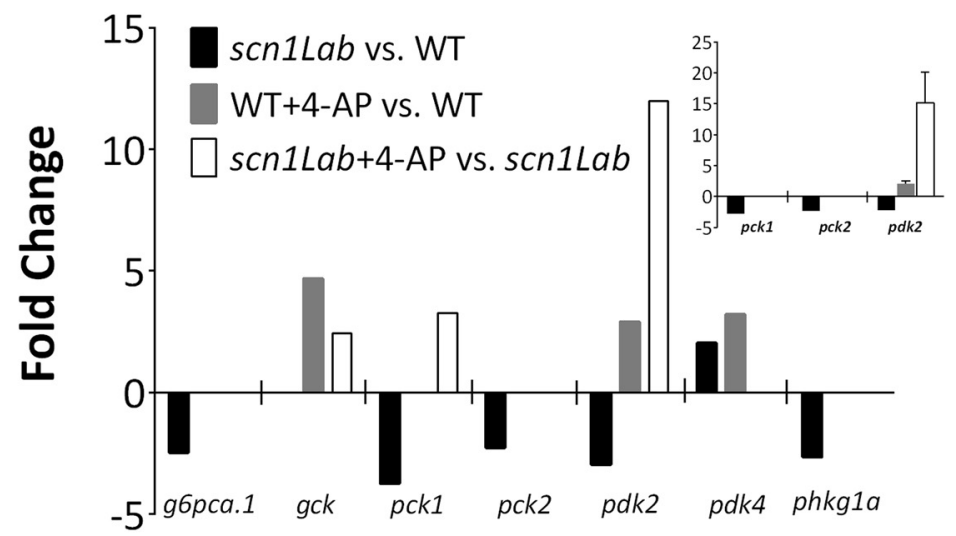

Figure 3. Glucose metabolism-related gene expression in WT and scn1Lab mutant zebrafish (DRVT) at baseline and after 4-AP stimulation. $\boldsymbol{A}$, Heatmap demonstrating relative expression of all genes analyzed from array. $\boldsymbol{B}$, Schematic depicting the pathways in which up or downregulated genes are involved. Red color indicates downregulated genes in scn1Lab mutant zebrafish vs WT at baseline. $\boldsymbol{C}$, Graph showing the seven genes with twofold or greater changes relative to respective controls. $n=6$ pooled embryos per group analyzed once. Inset, PCR verification of genes for which specific primers were available, pck2, pck4, and pdk2. $N=3$ groups of pooled embryos (6 per group) analyzed in triplicate, mean \pm S.E.M. 
A

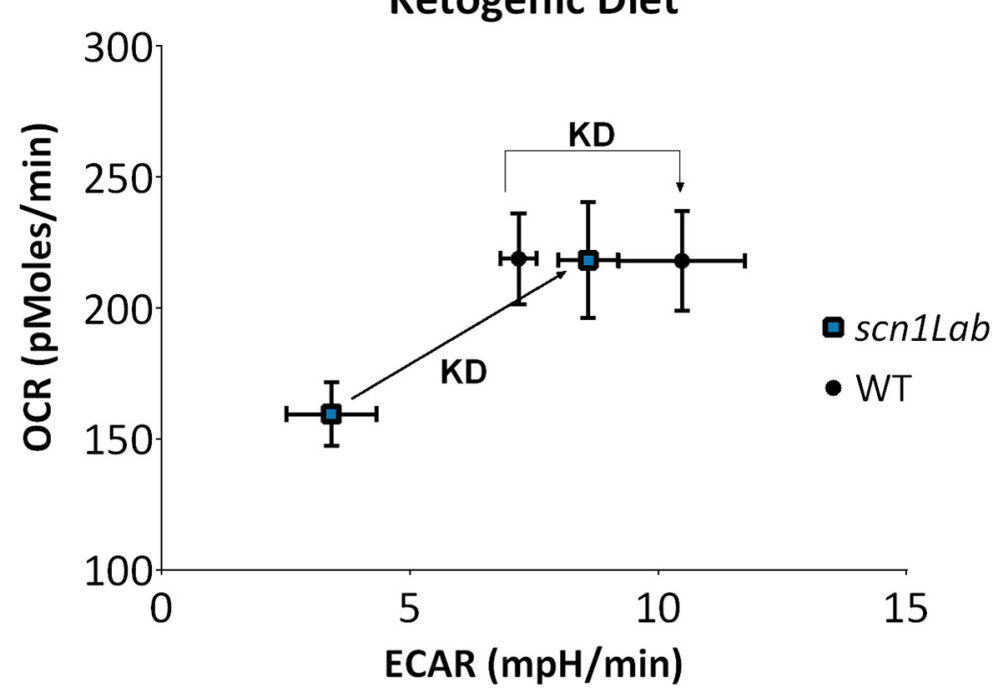

B Relative Glycolysis After 4-AP

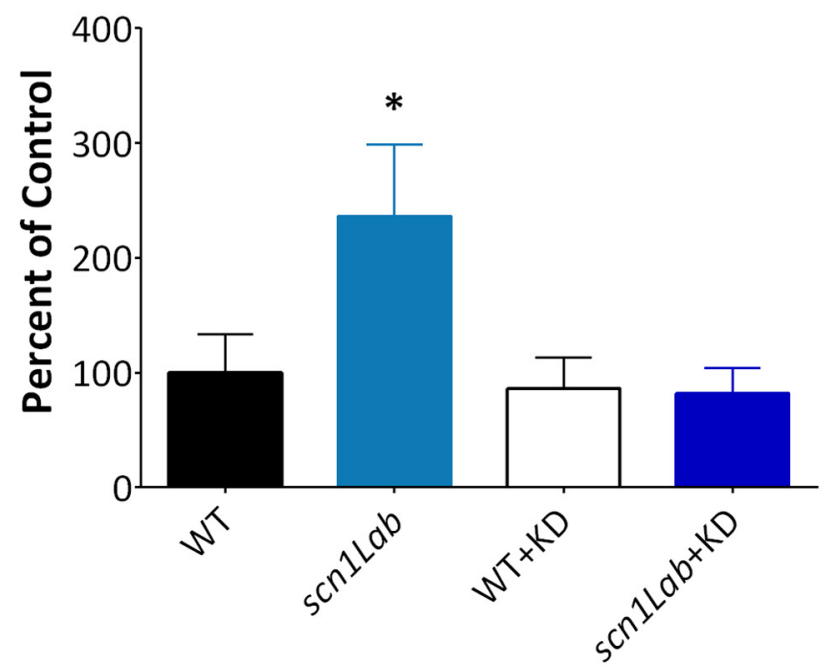

Figure 4. Ketogenic diet restores metabolism of scn1Lab mutant zebrafish to WT levels. A, Metabolic profile of WT zebrafish is shifted slightly to be more glycolytic after KD treatment. Mutant zebrafish increase both glycolysis and mitochondrial respiration to WT levels. $\boldsymbol{B}, \mathrm{KD}$ treatment reduces mutant zebrafish response to $4-\mathrm{AP}$ to similar to WT levels. $N=4$ (WT), 4 (scn1Lab), 5 (WT+KD), 5 $\left(\right.$ scn1Lab+KD), $p=.017^{\mathrm{jj}}$. Values or bars indicate mean \pm S.E.M.

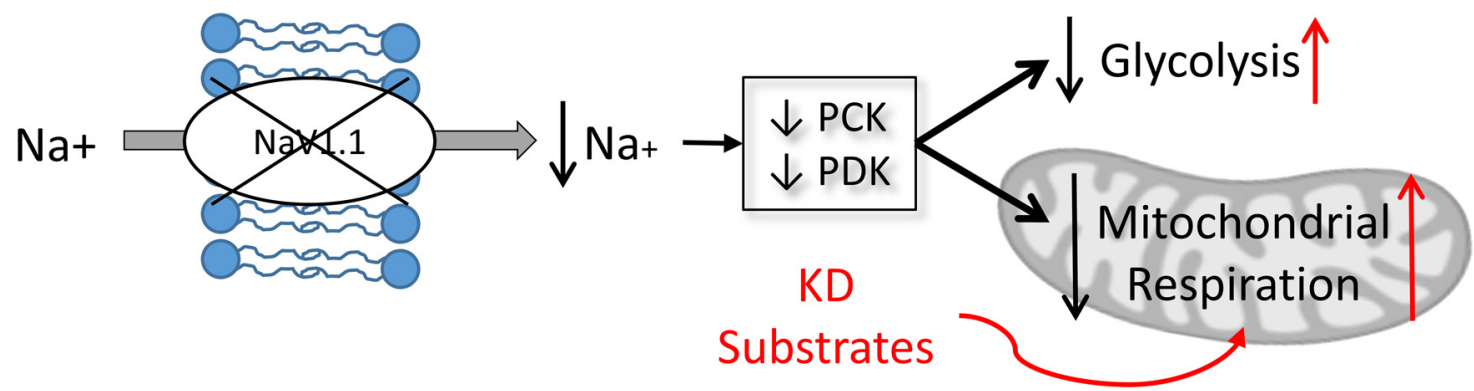

Figure 5. Summary diagram depicting proposed mechanism. Mechanism demonstrating changes in glycolysis and mitochondrial respiration in scn1 mutant zebrafish at baseline (in black) and proposed action of KD (in red). 
A

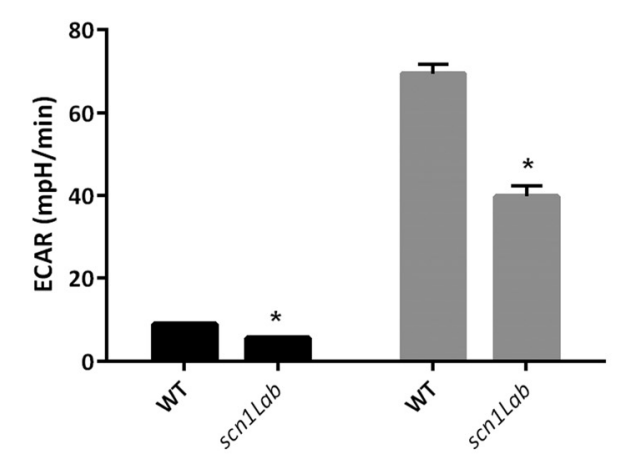

C

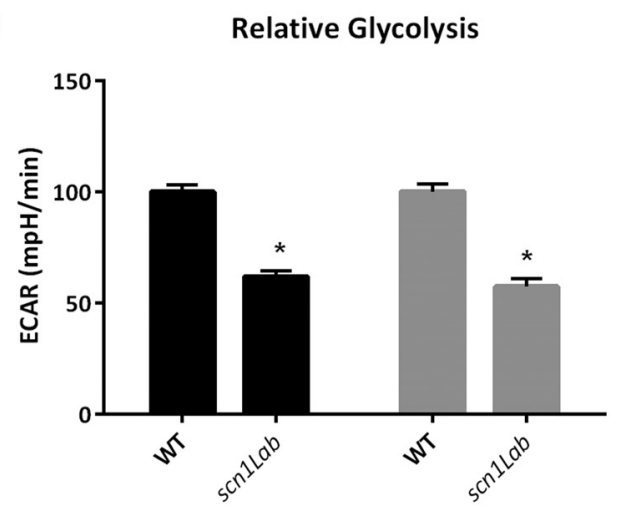

B
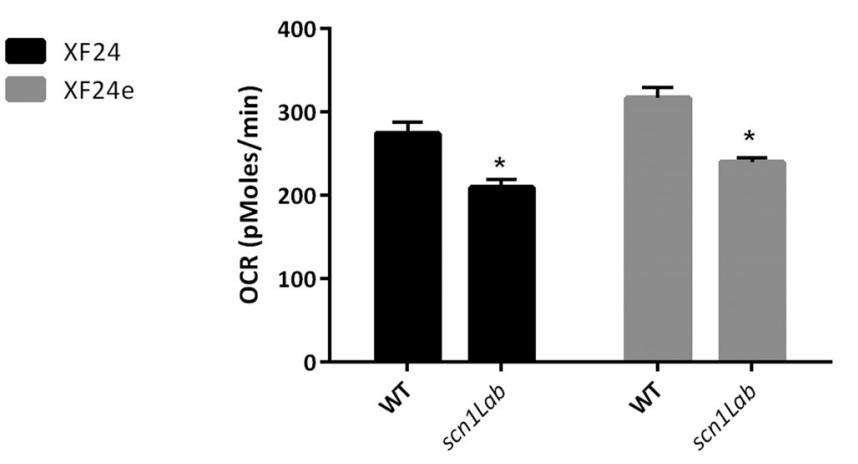

D

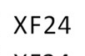
$\mathrm{XF24e}$

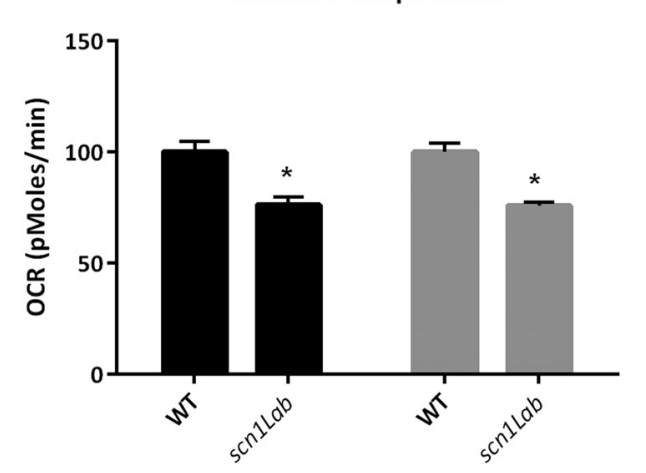

Figure 6. Metabolic differences in WT and scn1lab mutant zebrafish are reproducible when grown in a separate facility, as well as with different instrumentation. All zebrafish in this figure were bred and grown at UCD. Using the same instrumentation as Figures 1 and 4 (XF24) the baseline differences are recapitulated in zebrafish grown at UCSF. The newer model of extracellular flux analysis (XF24e) is more sensitive and thus has higher baseline values for both glycolysis and mitochondrial respiration. $\boldsymbol{A}, \boldsymbol{B}$, Absolute baseline differences in glycolysis and respiration are recapitulated in both the XF24 and newer XF24e. A, XF24, $p<.000^{\mathrm{kk}}$; XF24e, $p<.00^{\mathrm{Il}}$; $\boldsymbol{B}, \mathrm{XF24}, p=.00^{\mathrm{mm}} ; \mathrm{XF24e}, p<.00^{\mathrm{nn}} . \boldsymbol{C}, \boldsymbol{D}$, Although the XF24e is more sensitive, the relative differences in glycolysis and respiration are similar in both the XF24 and XF24e. C, XF24, $p<.00^{\circ 0}$; XF24e, $p<.00^{\mathrm{pp}}$. D, XF24, $p=.0002^{\mathrm{qq}} ; \mathrm{XF}^{24 \mathrm{e}}, p<.0001^{\mathrm{rr}}$. Bars represent mean \pm S.E.M.

sterer and Zarrouk Mahjoub, 2012). In addition, mitochondrial complex I deficiency has been observed in human and experimental temporal lobe epilepsy (Kunz et al., 2000). Whether the presence of seizures alone explains the metabolic deficits is unclear and needs further exploration.

Interestingly and consistent with a metabolic defect, KDs have shown efficacy in controlling seizures in some DS patients (Caraballo, 2011) and the scn1Lab mutant zebrafish used here (Baraban et al., 2013). We now show that a KD also improves the metabolic state of scn1Lab mutant zebrafish to WT levels. One potential mechanism of action is that the KD alters expression of glucoserelated metabolism genes in scn1 Lab mutant zebrafish to WT levels. However, this seems unlikely because alteration of the genes reported here, and others, by KD has not been reported in the literature. Murata et al. (2013) fed WT and Fgf21 (fibroblast growth factor) knockout mice KD and showed no difference in pck expression, or glucose6 -phosphatase and PPARy coactivator-1 $\alpha$, compared with normal diet. Jornayvaz et al. (2010) showed similar results in KD fed mice for pck, glucose-6-phosphatase, and pyruvate carboxylase compared to control diet. How- ever, a more likely mechanism is that a KD alters the fuels available for glycolysis and oxidative phosphorylation to use. Impairment of mitochondrial bioenergetics capacity can critically affect apoptosis, neuronal excitability, and seizure susceptibility (Gano et al., 2014). Ketone bodies can decrease the spontaneous firing of GABAergic neurons, dependent on $\mathrm{K}_{\text {ATP }}$ channels (Ma et al., 2007) and given the deficits in interneuron function thought to be associated with scn1a mutation this could be a direct site of action.

4-AP is a potassium channel blocker that is commonly used to elicit seizures in animal models, including larval zebrafish (Baraban et al., 2007). Here, we demonstrate that WT zebrafish respond to 4-AP by immediately increasing glycolysis but not mitochondrial respiration corresponding with severe behavioral seizures. The metabolic alteration is reminiscent of ictal hypermetabolism (Stafstrom et al., 2008) and it is interesting to note that scn1Lab mutants have delayed increases in glycolysis and mitochondrial respiration after a 4-AP challenge. We also report a significant downregulation of phosphoenolpyruvate carboxykinases (pck1 and pck2) and pyruvate dehydrogenase kinase (pdk2) in scn1Lab mutant, genes 
important in gluconeogenesis, glycogenolysis, and regulation of pyruvate dehydrogenase. Specifically, pck is responsible for converting oxaloacetate in to phosphoenolpyruvate, a glycolysis intermediary and its loss would result in less intermediates and less glycolytic flux. Pdk2 is a gene responsible for inhibition of pyruvate dehydrogenase, which converts pyruvate to acetyl CoA. Decreased pdk2 would result in increased pyruvate dehydrogenase activity and less pyruvate and lactate. This could explain the lower ECAR observed here (Sun et al., 2011). It may appear that increased pyruvate dehydrogenase would result in increased respiration in scn1Lab mutant zebrafish. However, because there are fewer intermediates, and less flux through glycolysis, it is reasonable that the overall respiration rate is reduced in scn1Lab mutant zebrafish. In WT zebrafish, the increase in pdk2 would increase inhibition of pyruvate dehydrogenase and subsequently lead to increases in glycolytic metabolites pyruvate and lactate, suggesting a mechanism for increased glycolysis.

Overall, our results suggest a metabolic impairment concomitant with a sodium channel mutation in DS, likely due to glycolysis gene-expression changes, and reversible by the KD (Fig. 5). We demonstrate this with a novel technique to assess ECAR and OCR in live zebrafish and propose that the metabolic impairment in scn1Lab mutant zebrafish may be due to changes in gene expression related to glucose metabolism (Fig. 3B). This approach was applied here to one genetic model of epilepsy, but opens new avenues for studying metabolism in any zebrafish model or as a means to screen compound libraries for new drugs that would improve metabolic function.

\section{References}

Artuso L, Romano A, Verri T, Domenichini A, Argenton F, Santorelli FM, Petruzzella V (2012) Mitochondrial DNA metabolism in early development of zebrafish (Danio rerio). Biochim Biophys Acta 1817:1002-1011. CrossRef Medline

Baraban SC, Taylor MR, Castro PA, Baier H (2005) Pentylenetetrazole induced changes in zebrafish behavior, neural activity and c-Fos expression. Neuroscience 131:759-768. CrossRef

Baraban SC, Dinday MT, Hortopan GA (2013) Drug screening in Scn1a zebrafish mutant identifies clemizole as a potential Dravet syndrome treatment. Nat Commun 4:2410. CrossRef Medline

Baraban SC, Dinday MT, Castro PA, Chege S, Guyenet S, Taylor MR (2007) A large-scale mutagenesis screen to identify seizureresistant zebrafish. Epilepsia 48:1151-1157. CrossRef Medline

Caraballo RH (2011) Nonpharmacologic treatments of Dravet syndrome: focus on the ketogenic diet. Epilepsia 52:79-82. CrossRef Medline

Chugani HT, Rintahaka PJ, Shewmon DA (1994) Ictal patterns of cerebral glucose utilization in children with epilepsy. Epilepsia 35:813-822. Medline

Craig AK, de Menezes MS, Saneto RP (2012) Dravet syndrome: patients with co-morbid SCN1A gene mutations and mitochondrial electron transport chain defects. Seizure 21:17-20. CrossRef

Dinday MT, Baraban SC (2015) Large-scale phenotype-based antiepileptic drug screening in a zebrafish model of Dravet syndrome $(1,2,3)$. eNeuro 2:ENEURO.0068-15.2015. CrossRef Medline

Dravet C (2011) The core Dravet syndrome phenotype. Epilepsia 52:3-9. CrossRef Medline

Finsterer J, Zarrouk Mahjoub S (2012) Epilepsy in mitochondrial disorders. Seizure 21:316-321. CrossRef Medline

Flynn JM, Choi SW, Day NU, Gerencser AA, Hubbard A, Melov S (2011) Impaired spare respiratory capacity in cortical synapto- somes from Sod2 null mice. Free Radic Biol Med 50:866-873. CrossRef Medline

Gano LB, Patel M, Rho JM (2014) Ketogenic diets, mitochondria, and neurological diseases. J Lipid Res 55:2211-2228. CrossRef Medline

Giménez-Cassina A, Martinez-François JR, Fisher JK, Szlyk B, Polak K, Wiwczar J, Tanner GR, Lutas A, Yellen G, Danial NN (2012) BAD-dependent regulation of fuel metabolism and K(ATP) channel activity confers resistance to epileptic seizures. Neuron 74:719730. CrossRef

Grisar T (1984) Glial and neuronal $\mathrm{Na}+-\mathrm{K}+$ pump in epilepsy. Ann Neurol 16:S128-S134. Medline

Hunter SE, Jung D, Di Giulio RT, Meyer JN (2010) The QPCR assay for analysis of mitochondrial DNA damage, repair, and relative copy number. Methods 51:444-451. CrossRef Medline

Jornayvaz FR, Jurczak MJ, Lee HY, Birkenfeld AL, Frederick DW, Zhang D, Zhang XM, Samuel VT, Shulman GI (2010) A high-fat, ketogenic diet causes hepatic insulin resistance in mice, despite increasing energy expenditure and preventing weight gain. Am J Physiol Endocrinol Metab 299:E808-E815. CrossRef Medline

Kunz WS, Kudin AP, Vielhaber S, Blümcke I, Zuschratter W, Schramm J, Beck H, Elger CE (2000) Mitochondrial complex I deficiency in the epileptic focus of patients with temporal lobe epilepsy. Ann Neurol 48:766-773. Medline

Lee EM, Park GY, Im KC, Kim ST, Woo CW, Chung JH, Kim KS, Kim JS, Shon YM, Kim YI, Kang JK (2012) Changes in glucose metabolism and metabolites during the epileptogenic process in the lithium-pilocarpine model of epilepsy. Epilepsia 53:860-869. CrossRef Medline

Liang LP, Waldbaum S, Rowley S, Huang TT, Day BJ, Patel M (2012) Mitochondrial oxidative stress and epilepsy in SOD2 deficient mice: attenuation by a lipophilic metalloporphyrin. Neurobiol Dis 45:1068-1076. CrossRef Medline

Ma W, Berg J, Yellen G (2007) Ketogenic diet metabolites reduce firing in central neurons by opening K(ATP) channels. J Neurosci 27:3618-3625. CrossRef Medline

Ma YY, Zhang XL, Wu TF, Liu YP, Wang Q, Zhang Y, Song JQ, Wang YJ, Yang YL (2011) Analysis of the mitochondrial complex I-V enzyme activities of peripheral leukocytes in oxidative phosphorylation disorders. J Child Neurol 26:974-979. CrossRef Medline

Mecocci P, MacGarvey U, Kaufman AE, Koontz D, Shoffner JM, Wallace DC, Beal MF (1993) Oxidative damage to mitochondrial DNA shows marked age-dependent increases in human brain. Ann Neurol 34:609-616. CrossRef Medline

Murata $\mathrm{Y}$, Nishio K, Mochiyama T, Konishi M, Shimada M, Ohta $\mathrm{H}$, Itoh N (2013) Fgf21 impairs adipocyte insulin sensitivity in mice fed a low-carbohydrate, high-fat ketogenic diet. PloS One 8:e69330. CrossRef Medline

Patel M, Day BJ, Crapo JD, Fridovich I, McNamara JO (1996) Requirement for superoxide in excitotoxic cell death. Neuron 16:345355. Medline

Rowley S, Liang LP, Fulton R, Shimizu T, Day B, Patel M (2015) Mitochondrial respiration deficits driven by reactive oxygen species in experimental temporal lobe epilepsy. Neurobiol Dis 75:151158. CrossRef Medline

Ryan K, Backos DS, Reigan P, Patel M (2012) Post-translational oxidative modification and inactivation of mitochondrial complex I in epileptogenesis. J Neurosci 32:11250-11258. CrossRef Medline

Stackley KD, Beeson CC, Rahn JJ, Chan SS (2011) Bioenergetic profiling of zebrafish embryonic development. PloS One 6:e25652. CrossRef Medline

Stafstrom CE, Roopra A, Sutula TP (2008) Seizure suppression via glycolysis inhibition with 2-deoxy-D-glucose (2DG). Epilepsia 49: 97-100. CrossRef Medline

Sun W, Chang SS, Fu Y, Liu Y, Califano JA (2011) Chronic CSE treatment induces the growth of normal oral keratinocytes via PDK2 upregulation, increased glycolysis and HIF1 $\alpha$ stabilization. Plos One 6:e16207. CrossRef Medline 
Taylor MR, Hurley JB, Van Epps HA, Brockerhoff SE (2004) A zebrafish model for pyruvate dehydrogenase deficiency: rescue of neurological dysfunction and embryonic lethality using a ketogenic diet. Proc Natl Acad Sci U S A 101:4584-4589. CrossRef Medline

Tenney JR, Rozhkov L, Horn P, Miles L, Miles MV (2014) Cerebral glucose hypometabolism is associated with mitochondrial dysfunction in patients with intractable epilepsy and cortical dysplasia. Epilepsia 55:1415-1422. CrossRef Medline

Tibbs GR, Barrie AP, Van Mieghem FJ, McMahon HT, Nicholls DG (1989) Repetitive action potentials in isolated nerve terminals in the presence of 4-aminopyridine: effects on cytosolic free $\mathrm{Ca} 2+$ and glutamate release. J Neurochem 53:1693-1699. Medline

Wallace DC, Zheng XX, Lott MT, Shoffner JM, Hodge JA, Kelley RI, Epstein CM, Hopkins LC (1988) Familial mitochondrial encephalomyopathy (MERRF): genetic, pathophysiological, and biochemical characterization of a mitochondrial DNA disease. Cell 55:601-610. Medline

Wu M (2009) Real-time measurement of mitochondrial respiration and glycolysis rates of cancer cells in a microplate AACR Education Book 2009 pp. 295-300. 